García, G., Gáfaro, A., Bermúdez, Y., Páez, D., Prada, E., Ruiz, F., Delgado, P., Rincón, G. (2016). Caracterización socioeconómica y vocacional de estudiantes de últimos grados de bachillerato en colegios públicos y privados: el caso del Área Metropolitana de Bucaramanga. Revista Lebret, 8. Bucaramanga, Colombia: Universidad Santo Tomás, pp. 265 - 289. ISSN: 2145-5996

\title{
Caracterización socioeconómica y vocacional de estudiantes de últimos grados de bachillerato en colegios públicos y privados: el caso del Área Metropolitana de Bucaramanga*
}

Vocational and socio-economic characterization of students in upper courses of public and private high schools: the case of the Wider Bucaramanga Area

Gustavo García Cediel', Aurora Inés Gáfaro Rojas ${ }^{2}, Y_{0}$ hana Bermudez ${ }^{3}$, Diana Páez Ardila ${ }^{4}$ Estefani Prada Villamizar ${ }^{5}$, Freddy Jesús Ruiz Herrera $^{6}$, Pedro Delgado $^{7}$, Guillermo Rincon ${ }^{8}$

\begin{abstract}
Resumen
Este trabajo tuvo como finalidad caracterizar socioeconómicamente y vocacionalmente a los jóvenes de una muestra piloto de colegios públicos y privados de once grado del Área Metropolitana de Bucaramanga, con el fin de orientar políticas públicas enfocadas en disminuir el desempleo juvenil, mejorar la pertinencia de la oferta educativa y fomentar mejores prácticas de orientación vocacional. Como metodología se planteó un trabajo de enfoque descriptivo mediante la aplicación de una encuesta (aplicativo web) suministrado por el Programa de las Naciones Unidas para el Desarrollo. Este trabajo se hizo en el marco de las investigaciones propuestas por el Observatorio Regional del Mercado de Trabajo - ORMET, de Santander con el patrocinio del Ministerio del Trabajo y el Programa de las Naciones Unidas para el Desarrollo.
\end{abstract}

Palabras clave

Desempleo juvenil, orientación vocacional.

Código de clasificación JEL: I21, L84, L89

* $\quad$ El artículo se deriva de un trabajo de investigación en el marco del Observatorio Regional de Mercado de Trabajo - ORMET, Santander, por parte de UNIRED con el apoyo del Programa de Naciones Unidas para el Desarrollo - PNUD y el Ministerio de Trabajo.

1 Magíster en Economía. Docente Universidad Cooperativa de Colombia. Vinculado al grupo de investigación Sectores de Clase Mundial - SECLAM. Correo electrónico: gustavo.garciac@campusucc.edu.co

2 Ph.D Estadística Matemática e Informática. Docente Salud Pública, Escuela de Medicina Universidad Industrial de Santander. Vinculada al Grupo de investigación en Demografía, Salud Pública y Sistema de Salud GUINDESS-UIS. Correo electrónico: agafaror@uis.edu.co

3 Doctora en Economía. Docente Facultad de Economía Universidad Santo Tomás Seccional Bucaramanga. Vinculada al grupo de investigación Economía Social y Desarrollo Empresarial. Correo electrónico: yoanapatriciabermudezgonzalez@ustabuca.edu.co

4 MSc Internacionalización Económica. Docente tiempo completo, Universidad Santo Tomás Seccional Bucaramanga. Vinculada al grupo de investigación Economía Social y Desarrollo Empresarial. Correo electrónico:diana.paez@ustabuca.edu.co

$5 \quad$ Magíster en Administración de Empresas. Correo electrónico: espravi@alumni.uv.es

6 MSc Ciencias Económicas, profesor Escuela de Economía y Administración, Universidad Industrial de Santander. Vinculado al grupo de investigación GIDROT. Correo electrónico: fjruizhe@uis.edu.co

7 Magíster en Ciencias Económicas. Magíster en Ciencia Política. Docente Universidad Autónoma de Bucaramanga - UNAB. Correo electrónico: pdelgado2@unab.edu.co

8 MBA en Administración de Empresas, docente investigador Universidad Pontificia Bolivariana Bucaramanga UPB. Correo electrónico: guillermo.rincon@upb.edu.co 


\begin{abstract}
This document aimed to characterize socioeconomically and vocationally young people in a pilot public and private schools BFA degree eleven sample in order to guide public policies aimed at reducing youth unemployment and fomenter best practices of vocational guidance. As work methodology descriptive approach was raised by the application of a survey (web application) provided by the United Nations Development Programme. This work was done within the framework of the research proposed by the Regional Labour Market Observatory, ORMET, Santander sponsored by the Ministry of Labor and the United Nations Development Programme.
\end{abstract}

\title{
Keywords
}

Youth unemployment, vocational guidance.

\section{Introducción}

La transición del sistema educativo al mercado laboral se convierte en una oportunidad y una amenaza para los sistemas económicos; es una oportunidad al definir oportunamente los perfiles laborales de la nueva mano de obra y el aporte que esta hace al crecimiento económico; a su vez es una amenaza pues, muchos jóvenes quedan por fuera del sistema engrosando el desempleo juvenil y dedicándose a tareas de baja productividad (Ramírez, 2002).

Teóricamente el desempleo estructural pretende explicar este fenómeno al definirlo como un desajuste entre las competencias necesarias para el trabajo que tienen los jóvenes y las que solicitan los empresarios (Campbell \& Stanley, 1997). En otras palabras una falta de concurrencia entre la oferta y la demanda laboral que muchas veces obedece a la falta de orientación vocacional que tienen los jóvenes. De esta manera preguntas como ¿Tienen los jóvenes condiciones claras frente a su selección de formación posterior a su Bachillerato?, ¿sus mecanismos de decisión están mediados por la moda o por el mercado o por sus intereses? necesitan respuestas para ser aplicadas las políticas públicas necesarias para subsanar esta situación.

Las discusiones sobre el desempleo juvenil están a la vanguardia en Latinoamérica, especialmente en ciudades intermedias como el Área Metropolitana de Bucaramanga (AMB en adelante) donde su configuración de mercado puede generar esquemas de exclusión natural, debido a fenómenos como informalidad y subempleo, pero también a condiciones de la demanda En este contexto, cobra importancia caracterizar socioeconómica y vocacionalmente a jóvenes del AMB, quienes pueden requerir un acompañamiento general a su experiencia de escogencia de posibles caminos de formación.

\section{Revisión de literatura}

El desempleo juvenil, dada su magnitud frente a tasas de otros grupos etarios, se ha convertido en un campo de estudio atractivo para la academia y de preocupación para los sectores gubernamentales que tratan de luchar contra este fenómeno, dado 
su impacto en la sociedad. Una de las aproximaciones al origen de esta problemática surge del concepto de desempleo estructural, pues su principal explicación es que no existe correspondencia entre la oferta y la demanda de mano de obra dado que los jóvenes no cuentan con las competencias necesarias para acceder a los puestos vacantes en el sistema económico (Campbell \& Stanley, 1997).

Entre las razones para esta falta de competencias se aducen varias. En primer lugar, las condiciones socioeconómicas afectan la situación laboral, pues según el ingreso del hogar, aquellos jóvenes pertenecientes a hogares de menores ingresos acumularan menores tasas de capital humano, lo que finalmente los lleva ser excluidos del sistema económico. (Burrows, 1998). El entorno familiar y las características ocupacionales de los padres inciden también en la situación laboral de los jóvenes pues sus padres contaran con menores redes e influencia, muchas veces determinante a la hora de encontrar trabajo (Coloma \& Vial, 2003).

De acuerdo con Weller (2006) y Guataqui, Forero \& García (2009) este desajuste entre la realidad del mercado laboral y las competencias de los jóvenes los llevan a trabajos de mala calidad o períodos muy largos de inactividad, lo que finalmente los obliga a retirarse del mercado laboral adoptando un estilo de vida titulado por Ullak \& Banks (1985) como el "vago de la esquina” o jóvenes ni-ni -ni estudian ni trabajan-y que muchas veces alimenta los fenómenos de pandillismo y drogadicción que se presentan.

Una de las herramientas planteadas para disminuir esta problemática es la orientación vocacional. Así, el constante e histórico reconocimiento de la orientación vocacional como una necesidad ligada a la obligación de educar para el trabajo y el desarrollo vocacional-profesional, es un tema que ocupa buena parte del desarrollo teórico en la educación, debido a la complejidad que representa no solo definir un concepto sino internalizarlo en la pedagogía y transmitirlo a la sociedad en tiempo presente. En la actualidad, el concepto de mayor uso institucional fue expuesto por la Revisión de Políticas de Orientación Profesional de la OCDE, la Comisión Europea y el Banco Mundial en 2004, expresado así:

La orientación profesional se refiere a los servicios y actividades destinados a ayudar a las persona, de cualquier edad y en cualquier punto a lo largo de sus vida para que la educación, la formación y opciones de empleo y gestionar sus carreras Estos servicios se pueden encontrar en las escuelas, las universidades y escuelas, en las instituciones de formación, en los servicios públicos de empleo, en el lugar de trabajo, en el sector voluntario o de la comunidad y en el sector privado. Las actividades pueden tener lugar de forma individual o en grupo, y pueden estar cara a cara oa distancia (incluyendo las líneas de ayuda y servicios basados en Internet). Ellos incluyen el suministro de información profesional (en la impresión, y otras formas basadas en las TIC), herramientas de evaluación y de autoevaluación, entrevistas de asesoramiento, programas de educación profesional (para ayudar a los individuos a desarrollar su conciencia de sí mismo, la conciencia de oportunidades y capacidades de orientación), 
programas de degustación (para probar las opciones antes de la elección de ellos), los programas de búsqueda de trabajo, y servicios de transición (OCDE y Comisión Europea, 2004, p. 10).

Sin embargo, este concepto ha vivido grandes transformaciones desde los inicios remotos de la orientación vocacional en 1275, cuando Ramón Llull redactó la Doctrina Pueril, un programa de educación en el que se presenta una descripción de profesiones para orientar a su hijo. El autor consideró que la educación debía contar con la diversidad de temperamentos y actitudes individuales, determinando de igual forma la elección de ocupaciones de acuerdo con la disposición natural; aunque su objetivo principal era hacer un aporte a la educación de los laicos, basada en los valores de la cristiandad combinados con el desarrollo profesional (Vilanou, 2013, p. 110).

En 1468, el teólogo Rodrigo Sánchez de Arévalo publicó su obra Speculum Vitae Humanae, un estudio pionero en descripciones ocupacionales en el que se resalta la importancia de la información profesional como punto de partida para la orientación vocacional. En contraste, Juan Luis Vives manifestó en su obra De tradendis disciplinis (1531) la necesidad de investigar las aptitudes individuales para conocerlas y enfocarlas a profesiones adecuadas. Esta corriente también fue sugerida por Juan Huarte de San Juan en su obra Examen de ingenio para la ciencia publicada en 1575, resaltando que no son solo las aptitudes sino también los ingenios o habilidades aptas y adecuadas que conectan a los individuos con las profesiones (Chacón, 2003, p. 32).

Sin embargo, no fue hasta 1909 cuando Frank Parsons en su publicación Choosing a vocation expone y desarrolla el concepto Vocational guidance (Orientación vocacional) y marca el inicio de la teorización y el estudio disciplinado de la orientación vocacional como una necesidad que debe ser explorada desde la academia y puesta a consideración para jóvenes y adultos. En principio, Parsons manifiesta que el problema de la orientación vocacional (visto así como una situación por resolver) debe ser resuelto a través de un método científico cuidadoso que tome en cuenta aptitudes, habilidades, ambiciones, recursos y limitaciones, así como la relación de estos elementos en su conjunto con las condiciones que delimitan el éxito en las diferentes industrias. La hipótesis planteada es que una ocupación que no esté en armonía con las aptitudes y capacidades de los trabajadores, desemboca en una ocupación ineficiente, sin entusiasmo y probablemente una ocupación desagradable y mal paga (Parsons, 1909, p. 3).

El autor es consciente sobre la dificultad que tienen los trabajadores de encajar sin inconvenientes en el mercado laboral y manifiesta que el cambio constante de empleos (a raíz de tomar decisiones aceleradas y sin justificación) explica un alto porcentaje de la ineficiencia laboral, así como altos costos en términos de contratación, despilfarro en los procesos de entrenamiento y servicios de baja calidad. Partiendo de ello, Parsons propone tres factores claves para solucionar el problema: 1) el autoanálisis 
(conocimiento de sí mismo, aptitudes, habilidades, intereses, ambiciones, recursos, limitaciones y sus causas), 2) información profesional del mercado laboral (requisitos y condiciones para alcanzar el éxito, ventajas y desventajas, compensaciones, oportunidades y prospectos en diferentes líneas de trabajo y, 3) conexión entre el sujeto y la profesión adecuada para su perfil. Para su ejecución, el autor propone tanto una amplia batería de preguntas como los discursos que a título personal recomienda para explicar la importancia de la orientación vocacional, su utilidad y la necesidad de explorar este campo junto a una persona profesional en orientación vocacional.

Gracias al trabajo de Parsons, se crea en 1913 la National Vocational Guidance Association (NUGA) a partir de la II Conferencia Nacional sobre Orientación Vocacional en los Estados Unidos. Hoy es conocida como National Career Development Association (NCDA).

Siguiendo los pasos de Parsons, Kelly (1914) publicó Educational Guidance, una tesis sobre el concepto Orientación Educativa que consiste en dar un carácter procesual a la orientación escolar, proporcionando ayuda al alumno en la elección de estudios y la solución a problemas de adaptación. Kelly identifica dos problemas neurálgicos para entender y probablemente alcanzar la eficiencia en la guía educacional: comprender la demanda de posibles tareas u ocupaciones y evaluar con cautela las habilidades en cada persona. Para ello, el autor propone un método completamente matemático: calcular la correlación entre lo que se estime como el nivel de ajuste entre una persona y una determinada actividad, a partir del sus intereses, aptitudes, gustos y su actuación en dicha actividad. Kelly rompe los esquemas en la orientación ocupacional y para sustentar su concepto propone evaluar los datos por medio de correlaciones parciales y ecuaciones de regresión, obteniendo con precisión el grado de capacidad individual frente a un curso superior o universitario (Kelly, 1914, p. 2).

En el ambito teórico conceptual de la orientación vocacional expuesto por Chacón (2003, p. 33) se resalta con precisión la postura de Álvarez (1994) para contextualizar el seguimiento a la orientación vocacional. En él, todos los conceptos son variables en la medida en que el contexto social, político y económico se modifique, generando a su vez procesos constantes de innovación educativa. Entre sus ejemplos se encuentra la transformación a partir de la Revolución Industrial y el desarrollo del sistema capitalista en su máxima expresión, así como los sistemas de comunicación e información lo hacen en el presente. En términos concretos, Chacón encuentra que para Álvarez son trascendentales los siguientes acontecimientos:

i. El movimiento de la Reforma Social que surge a partir del siglo XX en los países industrializados y en proceso de industrialización. Este fenómeno enmarcado en el modelo de producción de la sociedad generó consecuencias sociales tan fuertes que la estructura de la sociedad debió transformarse al mismo tiempo y reacomodarse a favor de la creciente industria, pagando costos muy altos en términos de bienestar. 
ii. El surgimiento de la psicotecnia como disciplina que busca adaptar el hombre al mercado laboral y a la selección de personal, surgen como consecuencia de la industrialización, en su afán por encontrar personas idóneas para desarrollar actividades determinadas para la industria.

iii. La psicometría apareció como un conjunto de métodos e instrumentos cuantificables usados para la descripción, investigación y comprobación de datos sobre el comportamiento psíquico. En otros términos, la psicometría se desarrolló como un método estadístico para caracterizar, en términos pedagógicos, a los individuos y de paso optimizar el sistema educativo a través de la selección de alumnos para garantizar el éxito en la transición al mercado laboral.

iv. El movimiento Pro Higiene Mental unido al Psicoanálisis son contemporáneos con el fortalecimiento de la orientación educativa y en conjunto fortalecieron el bien conocido "counseling" americano hasta posicionarlo en la cúspide de las políticas públicas en pro de la educación para el trabajo.

El counseling, una rama fundamental en la orientación ocupacional que está dirigida al ofrecimiento de información laboral en las escuelas y cuya aparición y desarrollo tuvo lugar entre 1930 y 1960; este fue influenciado por factores como el aumento de la población estudiantil a raíz de la abolición progresiva de la segregación racial y el acceso de la mujer, tanto a la educación universitaria como al mercado laboral. En este contexto, los servicios sociales empezaron a ocupar un lugar predominante entre las escuelas, como consecuencia de múltiples problemas generados por el crecimiento en la cantidad de estudiantes, hecho que determinó la aparición de la psicología escolar. En principio, el papel del orientador o psicólogo escolar estuvo delimitado por el proceso Diagnóstico-Pronóstico-Consejo, a partir de algunos test formulados con anterioridad sobre orientación vocacional; pero, gracias a la naturaleza educativa del proceso y su necesidad frente al mercado laboral, el counseling es una institución hasta el presente.

Con el tiempo, el desarrollo teórico de la orientación vocacional disminuyó su ritmo en cuanto a nuevos aportes esenciales aunque no su importancia y transformación. Por el contrario, gracias a los sistemas interactivos, la toma de decisiones vocacionales tiende a desarrollarse en gran parte a través de las nuevas tecnologías que ofertan una gran cantidad de opciones como información sobre distintas ocupaciones, comparación entre programas educativos, búsqueda y recepción de información sobre becas y ayudas para la formación, exploración libre en el campo de las opciones profesionales, identificación de habilidades requeridas para una ocupación, exploración de ocupaciones que se ajustan a los intereses y estilos de vida, apropiación en la toma de decisiones con el debido acompañamiento de orientadores, aprendizaje de estrategias para la búsqueda de empleo y reorientación profesional, entre muchas otras. 
Recientemente, González \& Lessire (2004) hicieron un aporte caracterizado por la necesidad de actualizar y recomponer la orientación vocacional frente a la diversidad de contextos globales, territoriales e individuales sin delimitaciones temporales. Para ellos, se debe partir de dos cambios importantes: el primero es una reconsideración ontológica en la realidad de la sociedad. "Esto implica para la Orientación, la creación de nuevos escenarios para la interacción interdisciplinaria y transdisciplinaria mediante una nueva dialéctica interdependiente" (González \& Lessire, 2004, p. 1). El segundo cambio está relacionado con el mercado laboral y de la educación: la necesidad de nuevas calificaciones, el comportamiento de la tasa de desempleo, subempleo e informalidad, nuevas organizaciones y nuevos trabajos, aumento en la matrícula escolar, nivel de demanda en los diferentes niveles de educación para todos los grupos por edad, nuevas demandas relacionadas con habilidades y conocimientos, y el uso del tiempo libre.

Krumboltz, citado por González y Lessire (2004, p. 2), expone unos postulados con el objetivo de redireccionar la orientación vocacional, en la conferencia inaugural del Congreso de la Association for Educational and Vocational Guidance (IAEVG) celebrada en Suiza (2003). Estos son:

- El objetivo de la orientación vocacional es crear una vida satisfactoria, no tomar una decisión vocacional.

- Los orientadores/as deben ayudar a los clientes a aprender cómo beneficiarse de las oportunidades o eventos fortuitos.

- Un trabajo es simplemente ayudar a otros. El éxito del mismo no es acumular riquezas ni lograr ocupar puestos gerenciales sino disfrutar el proceso.

- El objetivo de la jubilación no es ir a acostarse en la casa sino simplemente otra forma de seguir ayudando a las personas.

- En estos momentos más que pensar en la orientación vocacional tal como lo veníamos haciendo, deberíamos considerar una orientación para la transición (estudiante-persona-empleado-desempleado-jubilado).

- Se debe propiciar un mejor entrenamiento al futuro orientador/a.

- Debemos crear y capitalizar las oportunidades y eventos fortuitos que nos ocurren en la vida diaria.

\section{Metodología}

En el documento se utilizó una perspectiva descriptiva, a partir de datos cuantitativos obtenidos de una muestra de 10 colegios, donde se aplicó una encuesta a 305 jóvenes del AMB. Teniendo en cuenta que se conocía el valor de $\mathrm{N}$, la fórmula aplicada para hallar la muestra fue: 


$$
N=\frac{Z^{2} p q N}{E^{2}(N-1)+p q Z^{2}}
$$

La ficha técnica de la encuesta se presenta a continuación:

Ficha técnica de la encuesta

\begin{tabular}{ll}
\hline Universo: & $\begin{array}{l}\text { Jóvenes estudiantes de 10 y } 11 \text { grado de } \\
\text { colegios públicos y privados del AMB }\end{array}$ \\
\hline Error estimado: & $5 \%$ \\
Nivel de confianza: & $95 \%$ \\
Método de muestreo: & aleatorio simple \\
Fecha de realización de la encuesta: & Segundo semestre 2015 \\
Ciudad: & Área Metropolitana de Bucaramanga \\
\hline
\end{tabular}

Fuente: elaboración propia.

\section{Análisis de resultados}

\section{Caracterización socioeconómica}

Género-rango de edad: la siguiente figura permite identificar el número de jóvenes que participaron en la encuesta, distribuidos así:

\section{Figura 1. Género y edad}
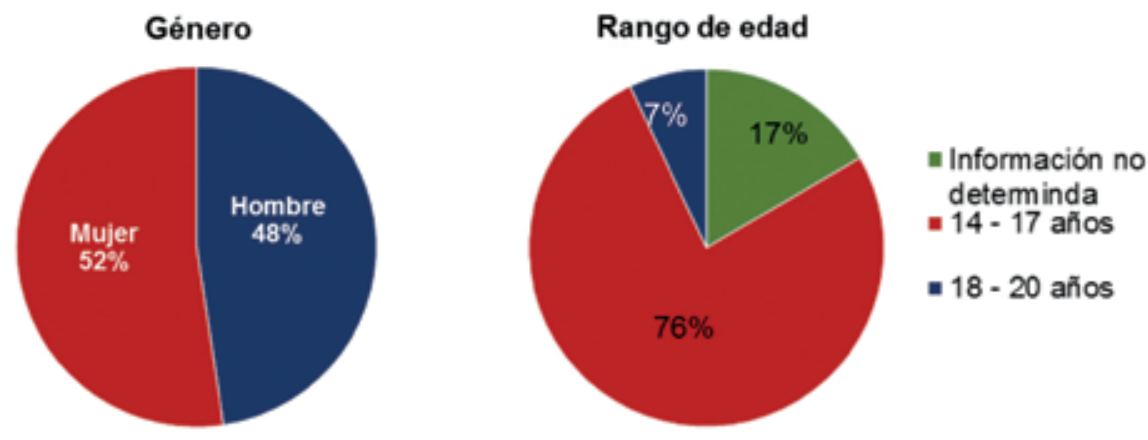

Fuente: elaboración propia con base en aplicativo web.

El género femenino obtiene el $52 \%$ de participación en esta encuesta. El $48 \%$ restante corresponde al género masculino. El mayor porcentaje de la población (76\%) se concentra en el rango de los 18 a 20 años de edad. 
Grado-institución educativa: 305 jóvenes (77\%) se encuentran cursando grado 11. 92 jóvenes $(23 \%)$ cursan grado 10 .

Figura 2. Grado institucion educativa

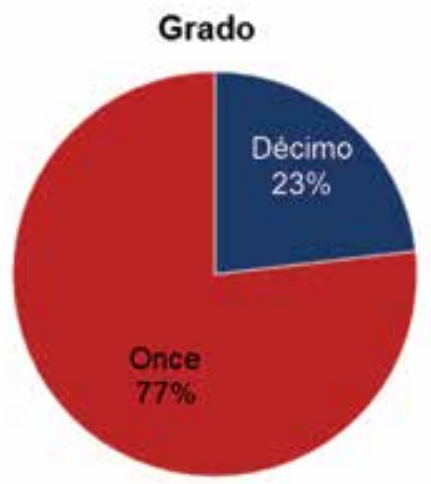

Fuente: elaboración propia.

Figura 3. Instituciones participantes en el piloto

\section{Nombre de la Institución}

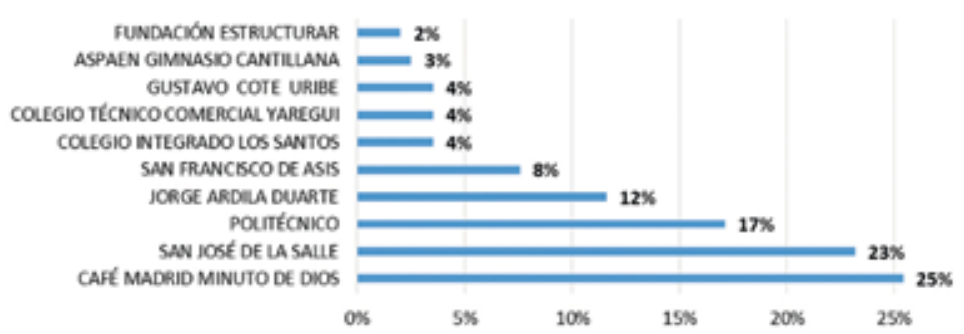

Fuente: elaboración propia.

Tabla 1. Personas por institución

\begin{tabular}{lc}
\hline \multicolumn{1}{c}{ Institución } & N. de personas por institución \\
\hline 1. Café Madrid Minuto de Dios & 101 \\
2. San José de La Salle & 92 \\
3. Politécnico & 68 \\
4. Jorge Ardila Duarte & 46 \\
5. San Francisco de Asis & 30
\end{tabular}




\begin{tabular}{lc} 
6. Colegio Integrado Los Santos & 14 \\
7. Colegio Técnico Comercial Yaregui & 14 \\
8. Gustavo Cote Uribe & 14 \\
9. Aspaen Gimnasio Cantillana & 10 \\
10. Fundación Estructurar & 8 \\
\hline Total general & $\mathbf{3 9 7}$ \\
\hline
\end{tabular}

Fuente: elaboración propia.

Población en condición de discapacidad: uno de los aspectos relevantes dentro de la caracterización es que indaga acerca de que la población que se encuentra en condición de discapacidad, para ese caso en particular, ocho (8) jóvenes registran información que corresponde a problemas de visión, alteraciones en el sistema cardiorespiratorio, sistema nervioso y movimiento del cuerpo.

Jóvenes con personas a cargo, específicamente con hijos

Figura 4. Estudiantes con hijos

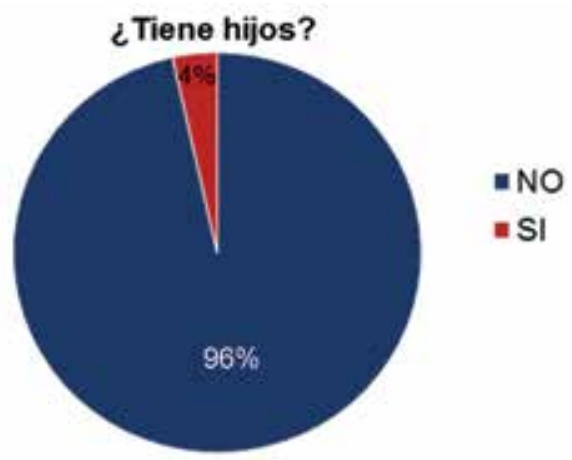

Fuente: elaboración propia.

Si bien, el porcentaje de jóvenes con hijos es bajo (14 estudiantes), con relación a la muestra total, es de vital importancia generar conciencia y acciones concretas desde el aula, que posibiliten la continuación de la formación educativa de estos jóvenes, con el fin de contribuir con su nivel de cualificación y por ende su nivel de vida.

Recursos ocupacionales de la población: este aspecto hace referencia a las redes productivas con los que cuentan los jóvenes o sus familias para la generación de ingresos. 
Figura 5. Negocios familiares
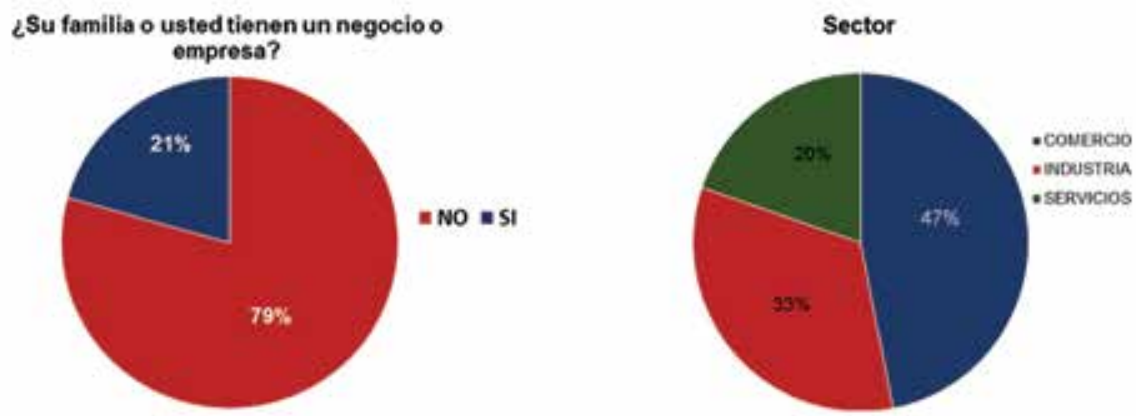

Fuente: elaboración propia.

La mayoría de negocios o unidades productivas con los que cuentan las familias corresponde al sector comercio. Entre ellos se encuentran negocios de venta de alimentos o prendas de vestir, peluquerías, talleres de mecánica, ferreterías, papelerías, fábricas de muebles y servicios inmobiliarios, entre otros.

La siguiente figura indaga acerca de los establecimientos educativos a los que han asistido los jóvenes a lo largo de su trayectoria escolar:

Figura 6. Número de establecimientos educativos asistidos

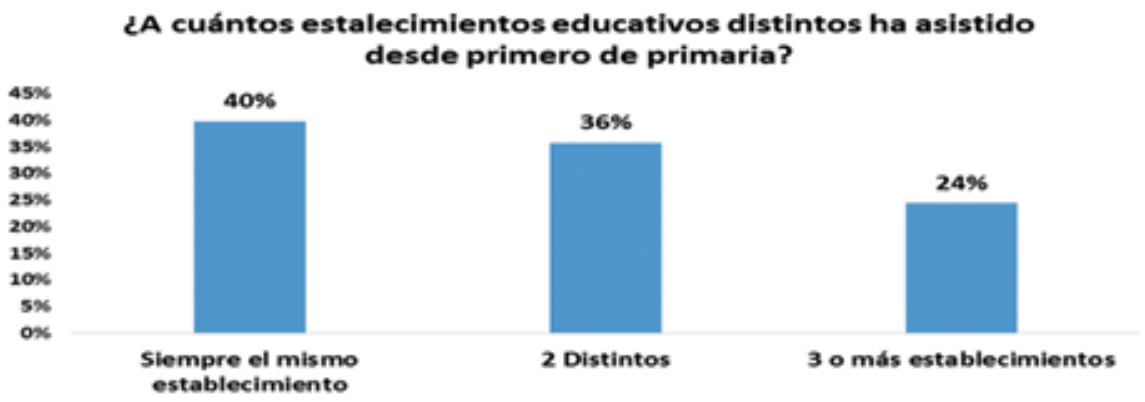

Fuente: elaboración propia.

El mayor porcentaje de la figura, $40 \%$, arroja que los estudiantes han asistido siempre al mismo establecimiento. 
Figura 7. Apoyo económico después de graduarse

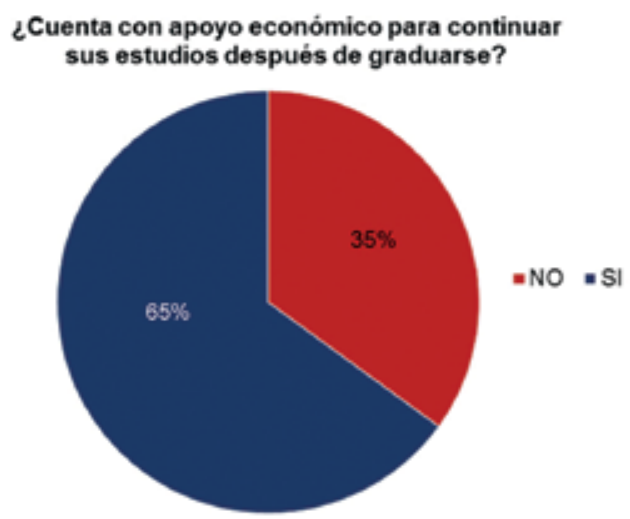

Fuente: elaboración propia.

Resultaría importante pensar qué estrategias se deben generar para facilitar la continuación de la formación académica para el $35 \%$ de la población que no cuenta con apoyo para seguir estudiando.

Líneas de especialización o énfasis de los colegios

Figura 8. Porcentaje de colegios con línea de profundización

\section{¿Tiene su colegio alguna especialidad o área de profundización?}

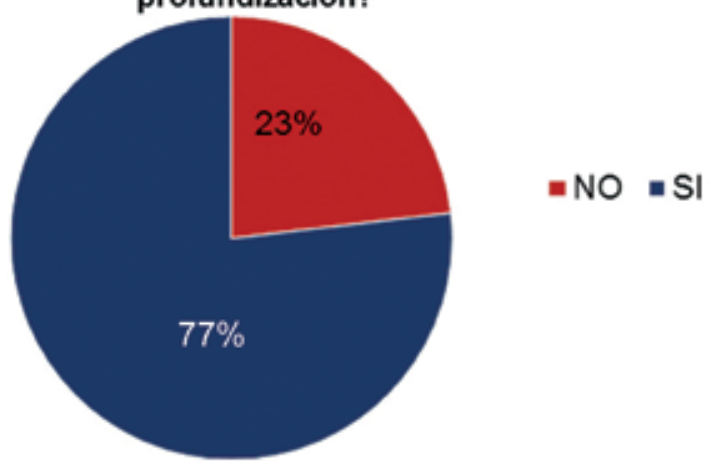

Fuente: elaboración propia. 
Figura 9. Líneas de profundización

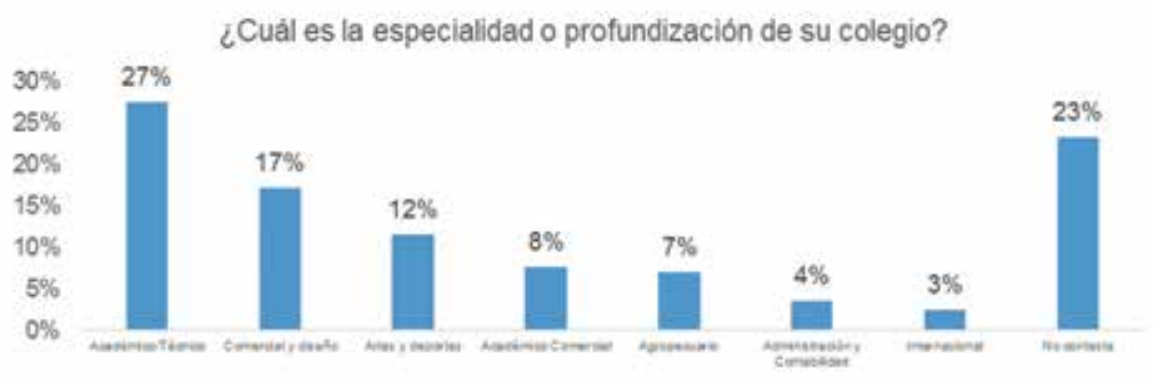

Fuente: elaboración propia.

Es importante conocer las líneas de especialización o profundización de los colegios, porque contribuyen con la definición del perfil vocacional y ocupacional de los jóvenes. Adicionalmente, les permiten desarrollar competencias de tipo técnico necesarias para acceder al mercado laboral.

\section{Bilingüismo en los estudiantes}

Figura 10. Porcentaje de bilingüismo

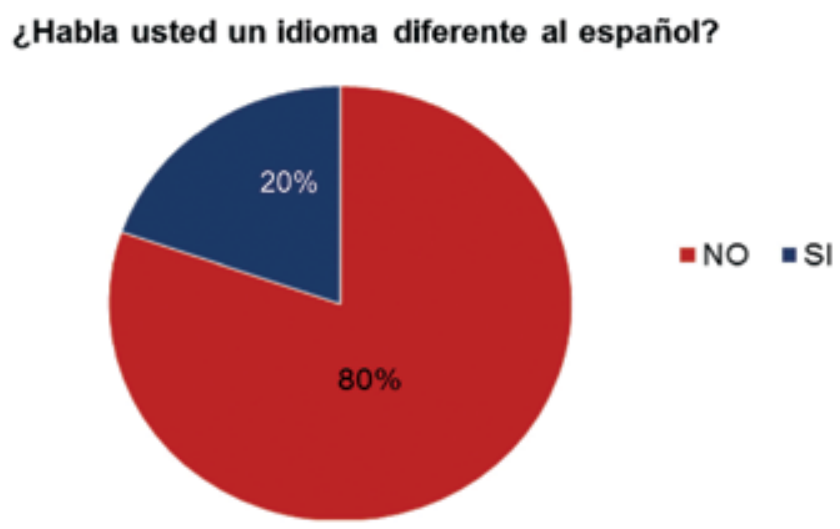

Fuente: elaboración propia.

Según la figura, un bajo porcentaje de los jóvenes hablan una segunda lengua. Es importante incentivar a los jóvenes para que accedan a formarse en otros idiomas y de igual manera, fortalecer y afianzar asignaturas de otras lenguas. 
Figura 11. Idioma hablado

¿Cuál idioma?

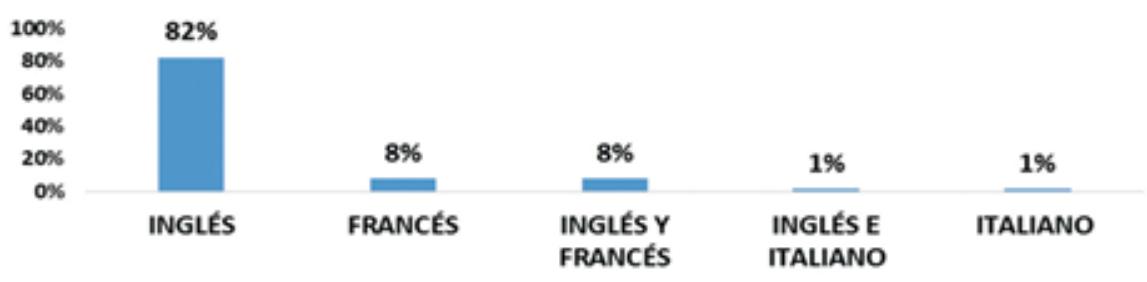

Fuente: elaboración propia.

El bilingüismo actualmente se constituye como una competencia de tipo personal y comunicativa que permite el acceso a mayor información, facilidades para el ingreso a universidades y cargos específicos. Así mismo, permite el intercambio con culturas que amplían la visión y perspectiva de futuro de los jóvenes.

\section{Áreas de capacitación}

Figura 12. Áreas de interés en capacitación

\section{¿En qué áreas le gustaría capacitarse?}

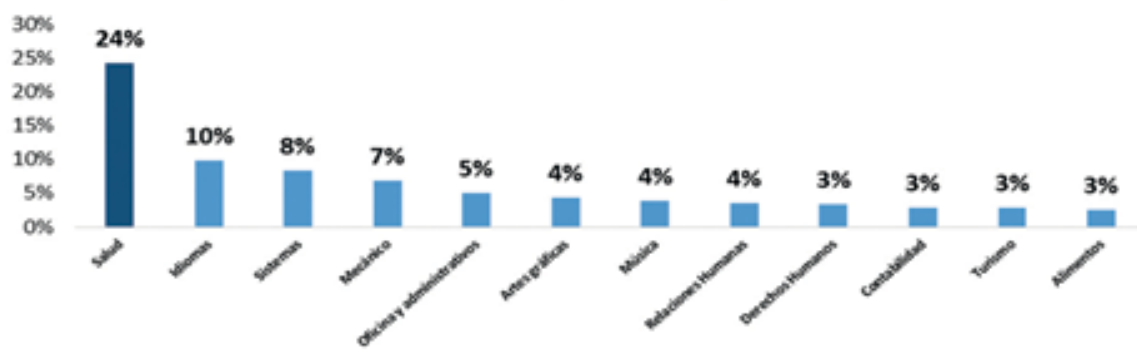

Fuente: elaboración propia.

El porcentaje más alto corresponde al 24\% para el área de salud. Medicina, enfermería, odontología, auxiliares de enfermería y odontología son algunas de las carreras elegidas. En segundo lugar con un 10\% al área de idiomas. Importante este porcentaje para continuar incentivando a los jóvenes en el aprendizaje de una segunda lengua. En tercer lugar con un $8 \%$ se encuentra el área de sistemas que se 
constituye como una competencia técnica de tipo transversal para desempeñarse en cualquier ámbito.

Figura 13. Carreras de mayor interés

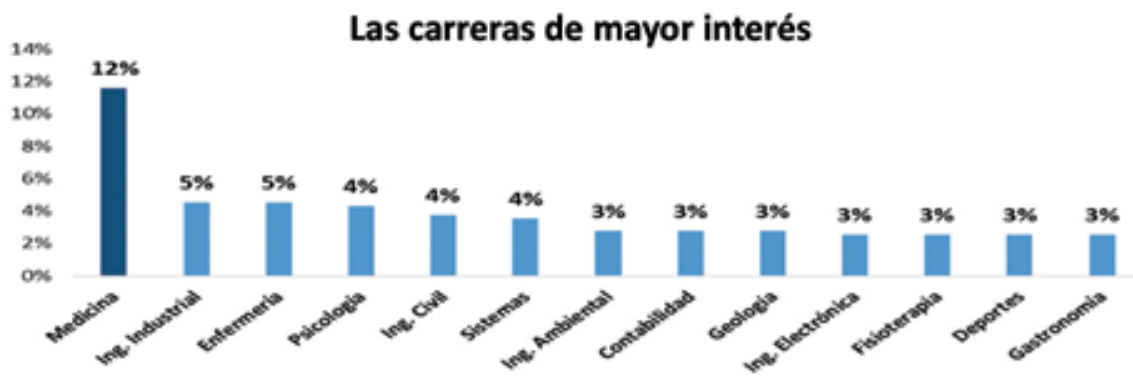

Fuente: elaboración propia.

Las áreas de conocimiento corresponden al observatorio de educación que estipula el Ministerio de Educación Nacional:

Figura 14. Áreas de conocimiento de mayor preferencia

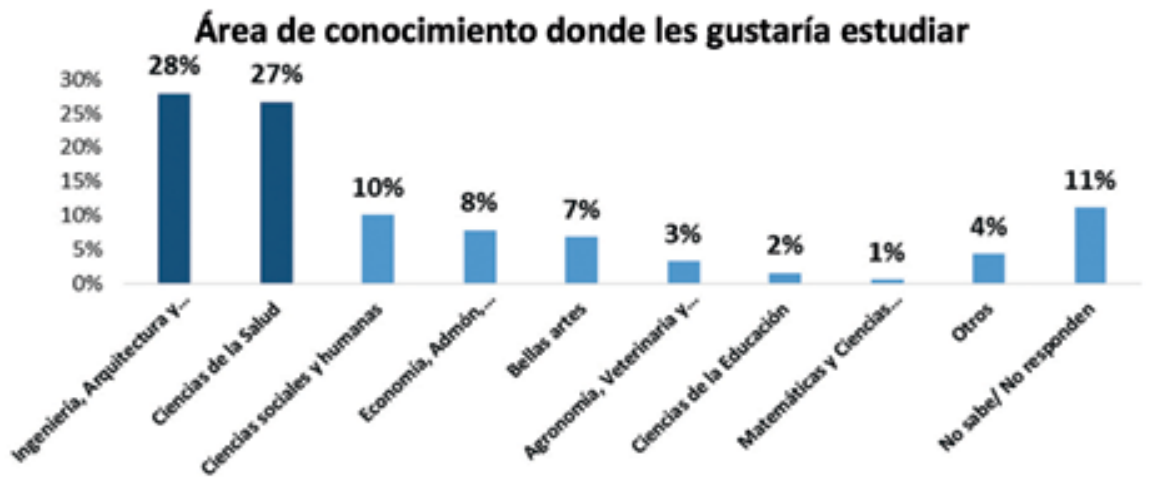

Fuente: elaboración propia.

Nivel de formación: la siguiente pregunta hace referencia a la elección de los jóvenes para alcanzar un nivel académico, esto bajo siete alternativas: 
Figura 15. Nivel educativo deseado

\section{¿A qué nivel educativo le gustaría llegar en el futuro?}

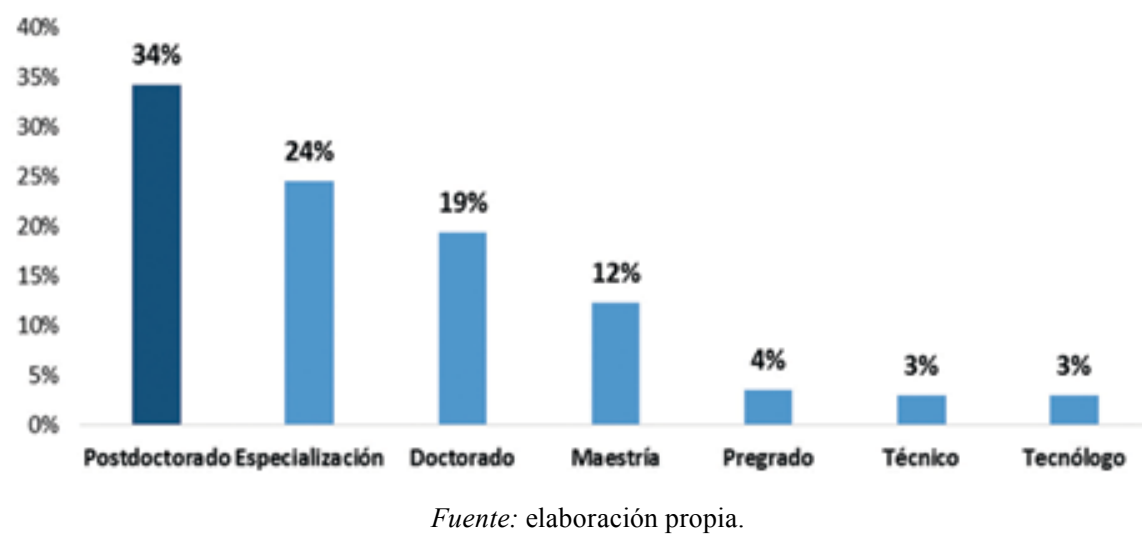

La figura permite observar que los porcentajes más altos de la población optan por los niveles de mayor cualificación educativa. Si bien, las aspiraciones y proyecciones de los jóvenes por profesionalizarse denotan el deseo por avanzar a un nivel de educación superior formal, sin ser esta garantía de oportunidades, es de vital importancia informarlos y concientizarlos acerca de las bondades y beneficios de una educación técnica o tecnológica; en la que, a través de ella se logra igualmente cualificar el potencial humano, y se facilita una formación más integrada hacia el sector productivo. Adicional a esto, responde oportunamente a las necesidades y realidades de algunos jóvenes: (entre ellas, su condición socioeconómica).

Figura 16. Mecanismo de financiación de estudios proyectado

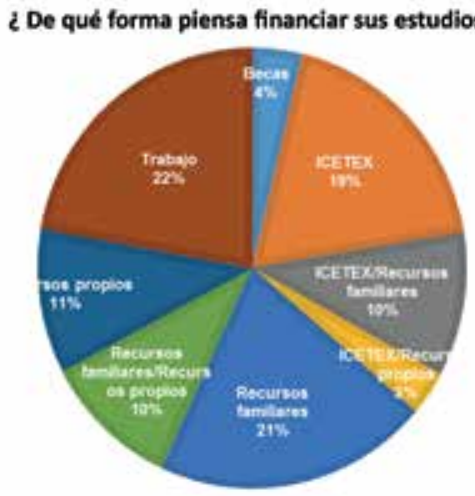

Fuente: elaboración propia. 
Trabajo: jóvenes que han trabajado.

Figura 17. Porcentaje de jóvenes que han trabajado

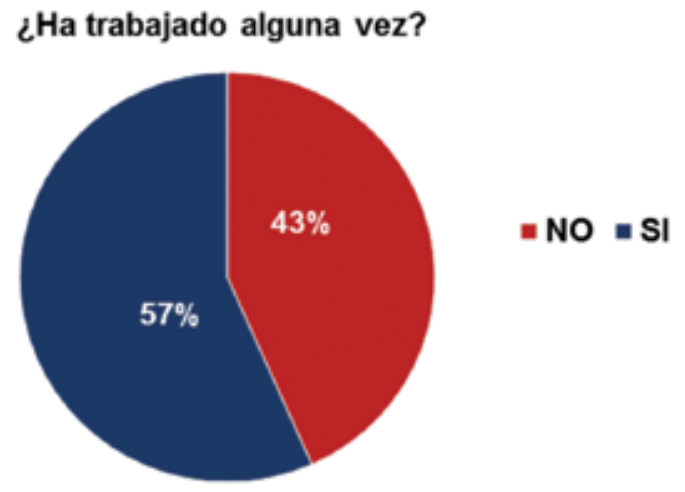

Fuente: elaboración propia.

El 43\% de la población, es decir, 172 jóvenes no ha tenido experiencia laboral. El $57 \%$ correspondiente a 225 jóvenes ha tenido experiencia laboral en los siguientes sectores así distribuidos:

Actividades en la que los jóvenes tienen experiencia

Tabla 2. Actividades en las que se cuenta con experiencia

\begin{tabular}{lcc}
\multicolumn{1}{c}{ Sector } & No. de estudiantes & $\%$ \\
\hline Ventas & 55 & $29 \%$ \\
Actividades artísticas y recreativas & 18 & $9 \%$ \\
Trabajo de oficina & 16 & $8 \%$ \\
Zapatería & 14 & $7 \%$ \\
Hotelería, turismo y restaurante & 7 & $4 \%$ \\
Mesero & 7 & $4 \%$ \\
Logística & 5 & $3 \%$ \\
Cajero & 4 & $2 \%$
\end{tabular}




\begin{tabular}{|c|c|c|}
\hline Comerciante & 4 & $2 \%$ \\
\hline Construcción & 4 & $2 \%$ \\
\hline Mensajería & 4 & $2 \%$ \\
\hline Belleza & 3 & $2 \%$ \\
\hline Carpintería & 3 & $2 \%$ \\
\hline Educación & 3 & $2 \%$ \\
\hline Tecnología & 3 & $2 \%$ \\
\hline Agricultura & 2 & $1 \%$ \\
\hline Confecciones & 2 & $1 \%$ \\
\hline Trabajo de cuidado a otros & 2 & $1 \%$ \\
\hline Bodega & 1 & $1 \%$ \\
\hline Conductor & 1 & $1 \%$ \\
\hline Llevando domicilios & 1 & $1 \%$ \\
\hline Otros & 30 & $16 \%$ \\
\hline \multirow[t]{2}{*}{ Ninguna } & 3 & $2 \%$ \\
\hline & 192 & \\
\hline
\end{tabular}

Fuente: elaboración propia.

La mayoría de actividades desempeñadas por los jóvenes eran de tipo no calificado, que requerían de un entrenamiento básico y repetición de las tareas. Ejemplo de ellas: vendedores, ayudantes, auxiliares, operarios.

Tabla 3. Sectores en los que muestran interés para trabajar

\begin{tabular}{lcc}
\hline \multicolumn{1}{c}{ Actividad } & No. personas & \% \\
\hline Actividades de asistencia humana & 19 & $17 \%$ \\
Comercio & 13 & $12 \%$ \\
Mecánica & 10 & $9 \%$ \\
Trabajo de oficina & 10 & $9 \%$ \\
Tecnología & 7 & $6 \%$ \\
Ventas & 7 & $6 \%$ \\
Actividades artísticas y deportivas y recreativas & 6 & $5 \%$ \\
Servicio de hotelería y restaurante & 5 & $4 \%$
\end{tabular}




\begin{tabular}{lcc} 
Deporte & 4 & $4 \%$ \\
Aviación & 3 & $3 \%$ \\
Construcción & 2 & $2 \%$ \\
Educación & 2 & $2 \%$ \\
Confección & 1 & $1 \%$ \\
En algo que beneficie a los animales & 1 & $1 \%$ \\
Logística y bodega & 1 & $1 \%$ \\
Restaurante & 1 & $1 \%$ \\
Otros & 20 & $18 \%$ \\
& $\mathbf{1 1 2}$ & \\
Información no determinada & $\mathbf{2 8 5}$ & \\
\hline
\end{tabular}

Fuente: elaboración propia.

De acuerdo con los resultados de la tabla anterior, persiste la coherencia e interés de los estudiantes por trabajar en ámbitos de servicio social, así como su deseo de capacitarse en áreas de la misma rama.

Tabla 4. Proyecciones e intereses

\begin{tabular}{lc}
\hline \multicolumn{1}{c}{ Qué le gustaría hacer una vez termine su bachillerato } & No. personas \\
\hline a. Trabajar & 8 \\
a. Trabajar, b. Crear empresa/negocio & 1 \\
a. Trabajar, c. Estudiar & 105 \\
a. Trabajar, d. Hacer carrera militar (policía/fuerzas militares) & 4 \\
b. Crear empresa/negocio & 8 \\
b. Crear empresa/negocio, c. Estudiar & 22 \\
b. Crear empresa/negocio, d. Hacer carrera militar (policía/fuerzas militares) & 1 \\
c. Estudiar & 215 \\
c. Estudiar, d. Hacer carrera militar (policía/fuerzas militares) & 14 \\
c. Estudiar, e. Ninguna de las anteriores & 1 \\
d. Hacer carrera militar (policía/fuerzas militares) & 18 \\
Total general & $\mathbf{3 9 7}$ \\
\hline
\end{tabular}

Fuente: elaboración propia. 
Resultados de las pruebas

Intereses: la siguiente figura muestra la tendencia de los estudiantes hacia 10 tipos de interés. Las barras rojas son los intereses con mayores porcentajes de inclinación.

Figura 18. Áreas de interés

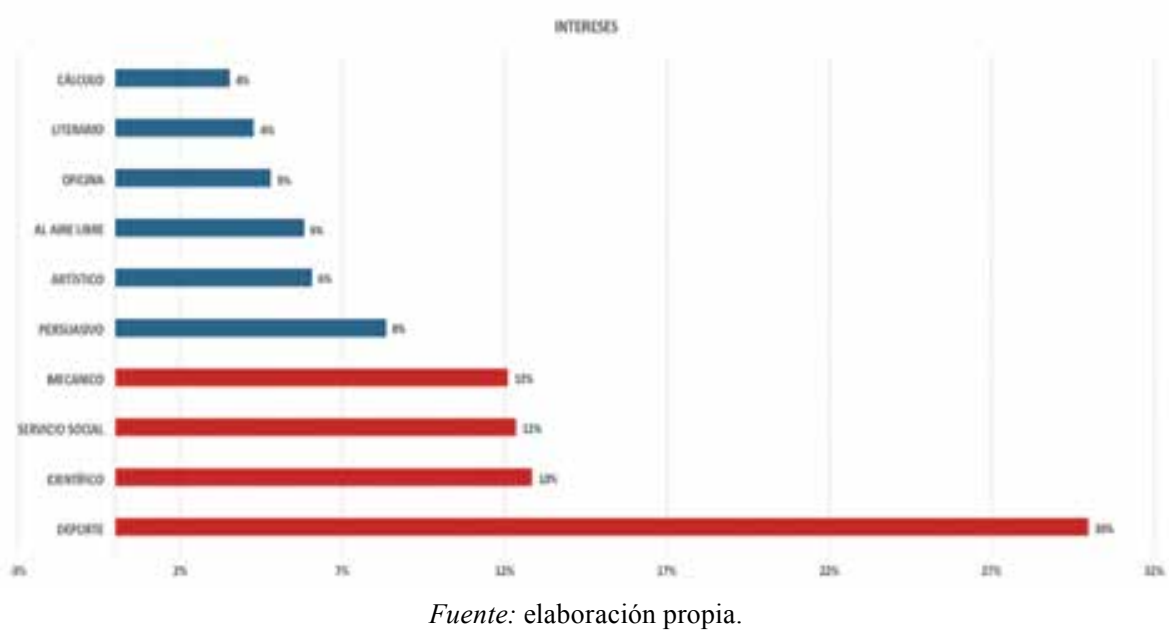

Dentro de los intereses más altos se encuentra el deportivo, con un 30\%. Este agrupa actividades que tienen que ver con prácticas deportivas, participación en competiciones, preparación o entrenamiento de diferentes deportes, o el gusto por mantener un bienestar físico. Al respecto, es importante anotar que en este caso, el interés deportivo puede surgir como un complemento paralelo a la carrera o actividad a la que se quiere dedicar el joven y que le permite complementar su vocación o gusto para el desarrollo pleno de su área profesional y de su vida personal. En segundo lugar, se encuentra el interés científico con un $13 \%$, el cual tiene que ver con actividades que se relacionan con la ciencia, el empleo del método científico, la utilización de conocimientos científicos en la industria y las investigaciones tendientes al descubrimiento de nuevos hechos. En tercer lugar, y con un 12\%, respectivamente, se encuentran los intereses de servicio social y el mecánico. El primero relacionado con actividades de ayuda a los demás y, el segundo, con actividades que implican trabajo con máquinas, herramientas, objetos mecánicos, aparatos eléctricos, etc. 
Se puede observar sincronía entre los resultados de la prueba de intereses y la pregunta de la caracterización que indaga sobre las áreas de conocimiento de mayor interés, así como de las carreras de mayor interés:

i. Las ingenierías: que corresponden al interés científico y mecánico.

ii. Las ciencias de la salud: que corresponden al interés por el servicio social.

La siguiente tabla establece algunas carreras o actividades que se circunscriben en los intereses de mayor inclinación en los jóvenes:

Tabla 5. Áreas de interés

\begin{tabular}{|c|c|}
\hline Tipo de interés & Carreras o actividades \\
\hline Interés deportivo & $\begin{array}{lrr}\text { Entrenador y } & \text { preparador } & \text { físico, } \\
\text { licenciado en } & \text { educación } & \text { física, } \\
\text { profesional en cualquier práctica } \\
\text { deportiva, } & \text { actividades r de } \\
\text { acondicionamiento físico, entre otras. }\end{array}$ \\
\hline Interés científico & $\begin{array}{l}\text { Ingenierías, arquitectos, físicos, } \\
\text { químicos, desarrollador de nuevos } \\
\text { productos, etc. }\end{array}$ \\
\hline Interés de servicio social & $\begin{array}{l}\text { Medicina, terapias en general, } \\
\text { odontología, enfermería, líderes por } \\
\text { la comunidad, sacerdotes, asistentes } \\
\text { sociales, etc. }\end{array}$ \\
\hline Interés mecánico & $\begin{array}{l}\text { Ingenierías, constructores, mecánicos, } \\
\text { ensambladores de piezas de } \\
\text { diferentes productos, reparadores de } \\
\text { instalaciones o aparatos eléctricos, } \\
\text { carpinteros, entre otros. }\end{array}$ \\
\hline
\end{tabular}

Fuente: elaboración propia.

Actitudes: la calificación de la prueba de actitudes está dada dentro de tres rangos: 4-5 denota que el estado de la actitud es óptima. 3 denota que las actitudes están en un nivel medio, el que se debe mejorar o fortalecer y el 1-2 corresponde a un nivel bajo: como lo muestra la figura siguiente. Las actitudes que se encuentran en las diferentes barras de color rojo responden a la calificación 1-2. Las de color amarillo corresponden a la calificación 3 y, el color verde corresponde a la calificación 4-5: 
Figura 19. Actitudes

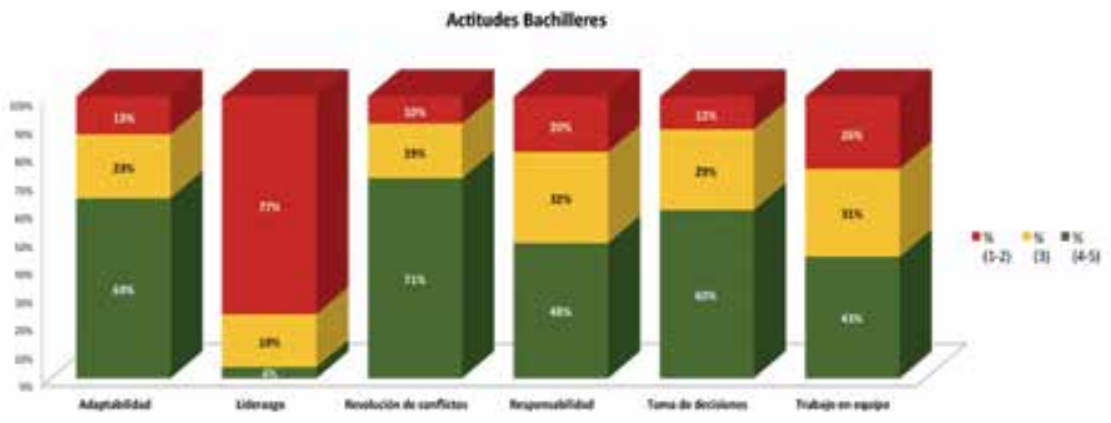

Fuente: elaboración propia.

Para este caso, la Adaptabilidad, la Resolución de conflictos y la Toma de decisiones se encuentran en un nivel 4-5: nivel de desarrollo óptimo.

Adaptabilidad: denota que la población tiene la capacidad para adaptarse a los cambios y de modificar su propia conducta para alcanzar objetivos. Denota facilidad y flexibilidad para adaptarse a situaciones nuevas y solucionar problemas de manera inmediata.

Resolución de conflictos: la población muestra indicios de asumir roles mediadores ante la existencia de un conflicto y de conducir las negociaciones de las partes para que este se resuelva.

Toma de decisiones: denota capacidad para tomar decisiones, identificar problemas, reconocer información significativa, analizar datos relevantes de una situación, diagnosticar posibles causas y aportar soluciones apropiadas y oportunas en el tiempo.

En nivel bajo1-2 se encuentran el Liderazgo y el Trabajo en equipo.

Liderazgo: reconocen que sus actitudes son importantes para liderar ideas y proyectos; sin embargo, debe ponerlas en práctica para fortalecer su capacidad de liderazgo.

Trabajo en equipo: se sugiere fortalecer la capacidad para trabajar en equipo permitiéndose desarrollar habilidades que contribuyan a mejorar el encuentro constructivo con los demás. Muestra actitudes para trabajar en equipo, escucha diferentes opiniones, pero en ocasiones se le dificulta aceptar las diferentes opiniones que se dan en el interior de un grupo, optando por trabajar con personas con las que tiene afinidad. 
Es fundamental tener en cuenta que las actitudes evaluadas son denominadas competencias blandas, las cuales hacen referencia a la competencia del SER, comportamientos y conductas de la persona que son necesarias para el desempeño dentro de contextos sociales y laborales. El desarrollo pleno de estas competencias se realiza a través de trabajo personal, talleres grupales o individuales con profesionales de las ciencias humanas-sociales.

Tendencia ocupacional: la prueba para esta tendencia tiene como objetivo evaluar cuatro habilidades relacionadas con las posibilidades que tiene una persona para generar ingresos: el empleo o el emprendimiento. Es así como se plantean y se evalúan 4 casos de la vida real y estos a su vez evalúan la planeación, asunción de riesgos, persistencia-resistencia al fracaso y la tendencia ocupacional, que hace referencia a la inclinación que tiene la persona hacia la empleabilidad o hacia el emprendimiento.

De acuerdo con los resultados de esta prueba, el 49,6\% de la población se inclina hacia la empleabilidad. Este resultado es congruente con las proyecciones de los jóvenes una vez terminen su bachillerato: "trabajar". Sin embargo, se debe tener en cuenta que es un porcentaje que se encuentra bordeando el siguiente nivel de la prueba que está dirigido hacia el emprendimiento básico o comerciante. Las personas con inclinación a la empleabilidad prefieren obtener una estabilidad y actividades que les sean impuestas. Prefieren no incurrir en riesgos y hacer carrera dentro de una empresa. La población que se inclina por el emprendimiento básico tiene la iniciativa para crear su propia idea de negocio pero debe reforzar habilidades de persistencia y resistencia al fracaso, planeación y asunción de riesgos; factores indispensables para iniciar un proceso de emprendimiento sostenible. Este es un aspecto que debe ser fortalecido con acompañamiento de profesionales idóneos y adicionalmente debería ser un aspecto importante para promocionar y potencializar en la población joven, para dinamizar el contexto productivo y económico del AMB.

\section{Conclusiones y recomendaciones}

En general, se evidencia un fuerte distanciamiento entre la exploración ocupacional en función del mercado laboral y toda actividad relacionada que se ejecute en el interior de las instituciones (en caso de existir tales actividades) si se tiene en cuenta que en su mayoría, los estudiantes poco diferencian la variedad de intereses y aunque en algunos existe una tendencia claramente delimitada (una buena cantidad de casos individuales), dentro de lo común en esta prueba piloto se observa que los intereses, en promedio, tienen niveles intermedios/altos muy cercanos entre sí.

En términos de la caracterización socioeconómica es posible afirmar que tanto el contexto que rodea los estudiantes en su vida familiar como académica tienen la capacidad de limitar o expandir la visión de futuro en los estudiantes. Existen comportamientos y tendencias casi que exclusivos en el interior de cada colegio en 
temas como el acceso a los medios de información, el interés por estudiar o trabajar, el acercamiento al mercado laboral en edad temprana, sus gustos e intereses, el nivel de bilingüismo o el salario que esperan recibir si trabajaran, entre otras variables.

En general, se observa que gran parte de los estudiantes encuestados desean estudiar en algún momento, ya sea a partir de cursos y capacitaciones o desde la educación formal. Esta conclusión es interesante a partir de la pregunta ¿A qué nivel educativo le gustaría llegar en el futuro?, en la que el 34\% respondió posdoctorado y casi en su totalidad se inclinó por niveles superiores al pregrado universitario. Aunque algunos de los estudiantes manifestaron no conocer este nivel, tienen muy claro que quieren lograr los retos más altos en términos académicos y, por ende, reconocen que la educación es fundamental para el desarrollo como seres humanos. En este sentido, es de suma importancia que se desarrollen esfuerzos (necesariamente amplios) desde la política pública y el sector privado para incentivar el desarrollo de intereses específicos que se enfoquen en el desarrollo productivo del área metropolitana.

En principio, es sorprendente y a la vez un motivo de preocupación que algunos colegios (representados por rectores, psicólogos o profesores con los que se tuvo contacto) se muestren apáticos frente a la exploración ocupacional de los estudiantes y consideren poco necesario y hasta una pérdida de tiempo el desarrollo de actividades que tienen como objetivo fortalecer la capacidad de tomar decisiones frente a la profesión a seleccionar y el lugar que deben ocupar las y los estudiantes en el mercado laboral una vez abandonen el colegio. Este comportamiento se puede apreciar en la cantidad de colegios que decidieron participar de la actividad, así como en el personal de algunas instituciones visitadas.

\section{Referencias}

Álvarez, V. (1994). Orientación educativa y acción orientadora. Madrid: EOS.

Burrows, S. (1998). Youth unemployment in the illawara region. Journal of Australian Political Economy, 28(32), 89-119.

Campbell, M., \& Stanley, B. (1997). Economía laboral contemporánea. Madrid: McGraw-Hill.

Chacón, O. (2003). Diseño, aplicación y evaluación de una propuesta de orientación vocacional para la educación media, diversificada y profesional venezolana. Universitat Rovira I Virgili, Programa de Doctorado: Innovación y Sistema Educativo.

Coloma, F., \& Vial, B. (2003). Desempleo e inactividad juvenil en Chile. Cuadernos de Economía, 150-171. 
González, J \& Lessire, O. (2004). Aspectos más recientes en orientación vocacional. Revista Iberoamericana de Educación 35(6).

Guataqui, J., Forero, N. \& Garcia, A. (2009). ¿A quiénes afecta el desempleo? Análisis de la tasa de incidencia. Lecturas de economía, 110-136.

Instituto Colombiano para el Fomento de la Educación Superior - ICFES. (1999). Grupo de la Evaluación de la Educación Básica y Media. Antecedentes y marco legal del examen de Estado.

Kelly, T. (1914). Educational guidance. Teachers college. Columbia University, 116.

Navajudas, F. (1998). Estrategias de enseñanza y aprendizaje en los centros rurales agrupados: análisis de una experiencia concreta para su innovación curricular. Universidad de La Rioja. Departamento de Ciencias Humanas y Sociales. Área de Pedagogía. Ediciones Universidad de Salamanca.

Parsons, F. (1909). Choosing a vocation. Boston: Houghton Mifflin

Ramírez, J. (2002). El desempleo juvenil, un problema estructural y global: el papel de las organizaciones de la sociedad civil. Tailandia: Ayuthaya.

Ullak, P., \& Banks, M. (1985): Youth unemployment and labour market withdrawal. Journal of Economic Psychology, 6, 51-64.

Vilanou, C. (2013). La doctrina pueril de Lulio: una enciclopedia escolar del siglo XIII. Educación XX1, 16(2), 97-114. Universidad Nacional de Educación a Distancia Madrid.

Weller, J. (2006). La inserción laboral de los jóvenes: características, tensiones y desafíos. Revista de la CEPAL, 92, 61-82. 